\title{
MHD Mixed Convection Micropolar Fluid Flow through a Rectangular Duct
}

\author{
Mekonnen Shiferaw Ayano $\mathbb{D}^{1},{ }^{1}$ Stephen T. Sikwila $\mathbb{D}^{2},{ }^{2}$ and Stanford Shateyi $\mathbb{D}^{3}$ \\ ${ }^{1}$ University of Swaziland, Private Bag 4, Kwaluseni, Swaziland \\ ${ }^{2}$ Sol Plaatje University, Private Bag X5008, Kimberley, South Africa \\ ${ }^{3}$ University of Venda, Private Bag X5050, Thohoyandou, South Africa
}

Correspondence should be addressed to Stanford Shateyi; stanford.shateyi@univen.ac.za

Received 5 July 2017; Revised 1 December 2017; Accepted 5 December 2017; Published 9 January 2018

Academic Editor: Filippo de Monte

Copyright (C) 2018 Mekonnen Shiferaw Ayano et al. This is an open access article distributed under the Creative Commons Attribution License, which permits unrestricted use, distribution, and reproduction in any medium, provided the original work is properly cited.

\begin{abstract}
Mixed convection flow through a rectangular duct with at least one of the sides of the walls of the rectangle being isothermal under the influence of transversely applied magnetic field has been analyzed numerically in this study. The governing differential equations of the problem have been transformed into a system of nondimensional differential equations and then solved numerically. The dimensionless velocity, microrotation components, and temperature profiles are displayed graphically showing the effects of various values of the parameters present in the problem. The results showed that the flow field is notably influenced by the considered parameters. It is found that increasing the aspect ratio increases flow reversal, commencement of the flow reversal is observed after some critical value, and the applied magnetic field increases the flow reversal in addition to flow retardation. The microrotation components flow in opposite direction; also it is found that one component of the microrotation will show no rotational effect around the center of the duct.
\end{abstract}

\section{Introduction}

The theory of fluids with microstructures has been the subject of a large number of investigations. These are realistic and important from a technological point of view. The classical theories of continuum mechanics are inadequate to explain the microscopic manifestations of complex hydrodynamic behaviour. Microcontinuum theory or generalized continuum theories incorporate independent deformations of the microstructure inside of a material point. There are a number of microcontinuum theories by Eringen $[1,2]$, namely, couple stress, micropolar, microstretch, and micromorphic. These theories impose more or less constraints on the motion of microstructure inside of a material point. In microstretch theory, it is assumed that the microstructure of each material point can undergo expansion or contraction independently in addition to translation and rigid rotation. This theory is a generalization of micropolar theory, in which the microstructure can only have translation and rigid rotation. Micromorphic theory constitutes extensions of the classical field theories concerned with the deformations, motions, and electromagnetic interactions of material media, as continua, in microscopic time and space scales. In micromorphic theory, a material body is considered as a continuous collection of deformable particles, each with finite size and inner structure. Axtell et al. [3] discussed blood circulation in tissues as a multistate, multiphase porous media problem by simulating the fluid phase as a micromorphic continuum. While blood is known to be Newtonian, it also has electrically conducting properties. Using the finite element method, Ansari et al. [4] studied micromorphic firstorder shear deformable plate element and the microstructure effect on the analysis of small-scale structures. Applications of micromorphic theory in micro-/nanoscale are discussed by formulating generalized micromorphic solid and fluid; see Lee and Wang [5]. Within the framework of micromorphic and elasticity theory, microstructure effect is developed to describe the bending behaviour of microplates by Ansari et al. [6]. Micropolar fluids are a subset of the micromorphic fluid theory introduced in a pioneering paper by Eringen [7]. 
Micropolar fluids constitute an important branch of nonNewtonian fluid dynamics where microrotation effects as well as microinertia are exhibited. Traditional Newtonian fluids cannot precisely describe the characteristic of fluid with suspended particles. Micropolar fluids formulated by Eringen [8] include certain microscopic effects arising from the local structure and micromotions of the fluid elements and provide a mathematical model for the non-Newtonian fluid flow behaviour such as exotic lubricants, polymers, animal blood, and real fluids with suspensions. Eringen [9] extended the micropolar fluid theory and developed the theory of thermomicropolar fluids. An excellent review of micropolar fluids and their applications was given by Ariman et al. [10]. Extensive review of foundations of the micropolar continuum mechanics is found in the book by Eremeyev et al. [11].

There have been numerous studies for the laminar flow and heat transfer through rectangular ducts [13-19]. Cheng and $\mathrm{Ou}$ [20] have also studied the mixed convection heat transfer in the thermal entrance region of horizontal rectangular channels. Aung and Worku [21] analyzed mixed convection flow in ducts with asymmetric wall heat fluxes. Mahaney et al. [22] studied development of laminar mixed convection flow in a horizontal rectangular duct with uniform bottom heating and Abou-Ellail and Morcos [23] also carried out investigations for similar flow geometry buoyancy effects in the entrance region. Huang et al. [24] examined laminar mixed convection heat and mass transfer in vertical rectangular ducts with film evaporation and condensation. Yan and Lin $[25,26]$ numerically investigated the laminar or turbulent mixed convection flow in a vertical channel under simultaneous influence of the combined buoyancy effects of the thermal and mass diffusion for an air-water vapour system.

To the knowledge of the authors and in all of the abovementioned studies, the combined effect of an applied magnetic field under strong buoyancy force on mixed convection on the micropolar fluid model which represents realistic non-Newtonian flow in a rectangular duct has not been well evaluated. This motivates the present investigation. In this paper, we study the problem of steady laminar, incompressible mixed convection micropolar fluid flow through a rectangular duct subject to applied magnetic field with at least one side of the rectangle being isothermal. Mathematical formulation has been presented for the governing equations and the solution is found numerically using the finite difference method. Results are discussed through velocity, microrotation, and temperature.

\section{Mathematical Formulation}

Consider a steady, laminar, incompressible, mixed convective heat transfer micropolar fluid through an infinite vertical rectangular duct given in Figure 1, with sides of length $a$ and $b$ in the presence of an external applied magnetic field. In the formulation of the problem, the following assumptions are made: Boussinesq approximations are valid, the flow is parallel and hence the only nonvanishing component is the

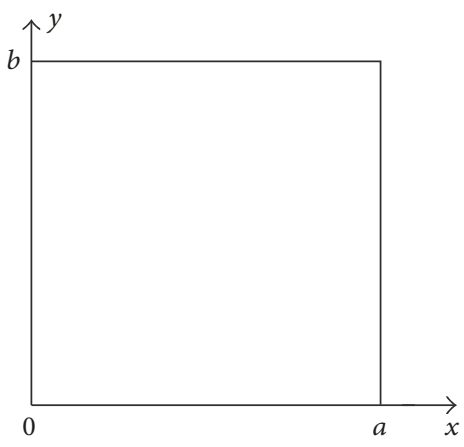

FIGURE 1: The base of the rectangular duct and the coordinate axes.

$Z$ (vertical) direction component, a uniform magnetic field is applied normal to the surfaces, and the induced magnetic field is assumed to be very small. The last assumption is considered to be valid for the case of small magnetic Reynolds number as done in previous works by Hayat et al. [27]. At least one of the duct walls is kept isothermal and the temperature is uniform along the duct vertical direction; hence $\partial T / \partial z=0$.

The equations of motion of a micropolar fluid by Eringen [8] are as follows:

$$
\begin{aligned}
& \nabla \cdot(\rho V)=0, \\
& (\lambda+2 \mu+\kappa) \nabla(\nabla \cdot \mathbf{V})-(\mu+\kappa) \nabla \times(\nabla \times \mathbf{V})+\kappa \nabla \times \phi \\
& \quad-\nabla P+\rho \mathbf{f}=\rho(\mathbf{V} \cdot \nabla) \mathbf{V}, \\
& (\alpha+\beta+\gamma) \nabla(\nabla \cdot \phi)-\gamma \nabla \times(\nabla \times \phi)+\kappa \nabla \times \mathbf{V}-2 \kappa \phi \\
& \quad+\rho \mathbf{I}=\rho j(\mathbf{V} \cdot \nabla) \phi, \\
& \alpha^{*} \nabla^{2} \mathbf{T}=\rho(\mathbf{V} \cdot \nabla) \mathbf{T},
\end{aligned}
$$

where $j$ is microinertia, $\mathbf{f}$ and $\mathbf{I}$ are body force and body couple per unit mass, $\alpha, \beta, \lambda, \mu$, and $\kappa$ are material constants (viscosity coefficients), $P$ is the thermodynamic pressure, $V=$ $(0,0, w)$ is velocity, $\phi=\left(\Gamma_{1}, \Gamma_{2}, 0\right)$ is microrotation, $\alpha^{*}$ is the thermal diffusivity, and $\mathbf{T}$ is the temperature of the fluid.

The constitutive equations, giving the stress tensor $\tau_{k l}$ and the couple stress tensor $M_{k l}$, are given by

$$
\begin{aligned}
\tau_{k l}= & \left(-P+\lambda v_{r ; r}\right) g_{k l}+\mu\left(v k ; l+v_{l ; k}\right) \\
& +\kappa\left(v_{l ; k}-\epsilon_{k l r} v_{r}\right) \\
M_{k l}= & \alpha v_{r ; r} g_{k ; l}+\gamma v_{l ; k},
\end{aligned}
$$

where $g_{k l}$ and $\epsilon_{k l r}$ are the metric tensor and the covariant $\epsilon$-symbol, respectively. The semicolon denotes the covariant partial differentiation with respect to a space coordinate and repeated indices are summed. The stress vector $\tau$ and couple stress vector $M$ at a point on a surface with normal $\mathbf{n}$ are given by $\tau_{\alpha k} \mathbf{n}_{\alpha}=\tau_{k}$ and $M_{\alpha k} \mathbf{n}_{\alpha}=M_{k}$. The material constants must 
satisfy the following inequalities, derived from the ClausiusDuhem inequality:

$$
\begin{gathered}
3 \lambda+2 \mu+\kappa \geq 0, \\
2 \mu+\kappa \geq 0, \\
\kappa \geq 0, \\
3 \alpha+\beta+\gamma \geq 0, \quad \gamma \geq|\beta| .
\end{gathered}
$$

The momentum, angular momentum, and energy equations may be written within boundary layer approximation as

$$
\begin{gathered}
\kappa\left(\frac{\partial \Gamma_{2}}{\partial x}-\frac{\partial \Gamma_{1}}{\partial y}\right)-P_{0}+\rho g\left[1-\beta_{T}\left(T-T_{0}\right)\right] \\
+(\mu+\kappa)\left(\frac{\partial^{2} w}{\partial x^{2}}+\frac{\partial^{2} w}{\partial y^{2}}\right)-\sigma B_{0}^{2} w=0 \\
-2 \kappa \Gamma_{1}+\kappa \frac{\partial w}{\partial y}-\gamma\left(\frac{\partial^{2} \Gamma_{2}}{\partial x \partial y}-\frac{\partial^{2} \Gamma_{1}}{\partial y^{2}}\right) \\
+(\alpha+\beta+\gamma)\left(\frac{\partial^{2} \Gamma_{1}}{\partial x^{2}}+\frac{\partial^{2} \Gamma_{2}}{\partial x \partial y}\right)=0 \\
-2 \kappa \Gamma_{2}-\kappa \frac{\partial w}{\partial x}+\gamma\left(\frac{\partial^{2} \Gamma_{2}}{\partial x^{2}}-\frac{\partial^{2} \Gamma_{1}}{\partial x \partial y}\right) \\
+(\alpha+\beta+\gamma)\left(\frac{\partial^{2} \Gamma_{1}}{\partial x \partial y}+\frac{\partial^{2} \Gamma_{2}}{\partial x^{2}}\right)=0 \\
\frac{\partial^{2} T}{\partial x^{2}}+\frac{\partial^{2} T}{\partial y^{2}}=0 \\
T_{0}=\int_{0}^{a} d x \int_{0}^{b} d y T
\end{gathered}
$$

where $w$ is flow velocity, $\Gamma_{1}$ and $\Gamma_{2}$ are components of microrotation, $T$ is the temperature, $\rho$ is fluid density, $\mu$ is dynamic coefficient of viscosity, $\kappa$ is vortex viscosity, $\alpha, \beta$, $\gamma$ are material constants (the spin, gradient, and viscosity), $g$ is the acceleration due to gravity, $\beta_{T}$ is the coefficient of thermal expansion, $T_{0}$ is the reference (mean) temperature, $B_{0}$ is magnetic induction, and $\sigma$ is electric conductivity of the fluid. We introduce the nondimensional variables

$$
\begin{aligned}
x & =\tilde{x} a, \\
y & =\tilde{y} a, \\
w & =\widetilde{w} U_{0}, \\
\Gamma_{1} & =\frac{v_{1} U_{0}}{a}, \\
\Gamma_{2} & =\frac{v_{2} U_{0}}{a}, \\
T-T_{0} & =\left(T_{1}-T_{2}\right) \theta, \\
\sigma & =\frac{b}{a}
\end{aligned}
$$

and the mean velocity

$$
U_{0}=\frac{1}{a b} \int_{0}^{a} d x \int_{0}^{b} w d y .
$$

Substituting the nondimensional variables in (4) and dropping the tildes, we get

$$
\begin{aligned}
& \frac{N}{1-N}\left(\frac{\partial v_{2}}{\partial x}-\frac{\partial v_{1}}{\partial y}\right)-\lambda+\frac{\mathrm{Gr}}{\mathrm{Re}} \theta \\
& +\frac{1}{1-N}\left(\frac{\partial^{2} w}{\partial x^{2}}+\frac{\partial^{2} w}{\partial y^{2}}\right)-H a^{2} w=0 \\
& -2 v_{1}+\frac{\partial w}{\partial y}-\frac{2-N}{m^{2}}\left(\frac{\partial^{2} v_{2}}{\partial x \partial y}-\frac{\partial^{2} v_{1}}{\partial y^{2}}\right) \\
& +\frac{1}{l^{2}}\left(\frac{\partial^{2} v_{1}}{\partial x^{2}}+\frac{\partial^{2} v_{2}}{\partial x \partial y}\right)=0 \\
& -2 v_{2}-\frac{\partial w}{\partial x}+\frac{2-N}{m^{2}}\left(\frac{\partial^{2} v_{2}}{\partial x^{2}}-\frac{\partial^{2} v_{1}}{\partial x \partial y}\right) \\
& +\frac{1}{l^{2}}\left(\frac{\partial^{2} v_{1}}{\partial x \partial y}+\frac{\partial^{2} v_{2}}{\partial y^{2}}\right)=0 \\
& \frac{\partial^{2} \theta}{\partial x^{2}}+\frac{\partial^{2} \theta}{\partial y^{2}}=0
\end{aligned}
$$

with the additional conditions

$$
\begin{aligned}
& \int_{0}^{1} d x \int_{0}^{y_{0}} d y \theta=0 \\
& \int_{0}^{a} d x \int_{0}^{y_{0}} w d y=y_{0}
\end{aligned}
$$

where $N=\kappa /(\mu+\kappa)$ is the coupling number, $l^{2}=2 a^{2} \kappa /(\alpha+$ $\beta+\gamma)$ is a nondimensional parameter, $\mathrm{Gr}=g \beta_{T}\left(T_{2}-T_{1}\right) d^{3} / \nu^{2}$ is the thermal Grashof number, $\operatorname{Re}=U_{o} d / \nu$ is the Reynolds number, $\mathrm{Ha}^{2}=\sigma B_{0}^{2} a^{2} / \rho v$ is the Hartman number, $v$ is the kinematic viscosity, $m^{2}=d^{2} \kappa(2 \mu+\kappa) / \gamma(\mu+\kappa)$ is micropolar parameter, $d=2 a b /(a+b)$ is the hydraulic diameter, and $\lambda=a^{2} P_{o} / \mu U_{o}$ is a dimensionless parameter.

The boundary conditions for velocity and microrotations in dimensionless form are as follows:

$$
\begin{aligned}
& w(x, 0)=w(x, \sigma)=w(0, y)=w(1, y)=0, \\
& v_{1}(x, 0)=v_{1}(x, \sigma)=v_{1}(0, y)=v_{1}(1, y)=0, \\
& v_{2}(x, 0)=v_{2}(x, \sigma)=v_{2}(0, y)=v_{2}(1, y)=0 .
\end{aligned}
$$

The thermal boundary conditions for two cases are set as follows. 
TABLE 1: Values of $w(x, y)$ for different positions in the duct.

\begin{tabular}{lcc}
\hline$(x, y)$ & Barletta [12] & Present \\
\hline$(0.5,0.5)$ & 0.073671 & 0.073560 \\
$(0.6,0.6)$ & 0.068744 & 0.068639 \\
$(0.75,0.75)$ & 0.045286 & 0.045206 \\
$(0.85,0.85)$ & 0.032666 & 0.023547 \\
$(0.9,0.9)$ & 0.013071 & 0.013026 \\
\hline
\end{tabular}

Case 1 (thermal boundary conditions).

$$
\begin{aligned}
\left.\frac{\partial T}{\partial x}\right|_{x=0} & =0, \\
T(x, 0, Z) & =T_{1}, \\
\left.\frac{\partial T}{\partial x}\right|_{x=a} & =0, \\
T(x, b, Z) & =T_{2} .
\end{aligned}
$$

Case 2 (thermal boundary conditions).

$$
\begin{gathered}
\left.k \frac{\partial T}{\partial y}\right|_{y=0}=-q_{w}, \\
T(0, y, z)=T_{1}, \\
\left.k \frac{\partial T}{\partial y}\right|_{y=b}=q_{w}, \\
T(a, y, z)=T_{1} .
\end{gathered}
$$

Applying the dimensionless parameters, taking the reference temperature difference $\Delta T=T_{1}-T_{2}$, and assuming $T_{1}>$ $T_{2}$ for the thermal boundary conditions (Case 1) and $\Delta T=$ $a\left(q_{w} / k\right)$ for the thermal boundary conditions (Case 2$)$, we get the dimensionless thermal boundary conditions as follows.

Two Isothermal and Two Adiabatic Walls. Surfaces parallel to the $y$-axis are assumed adiabatic and temperature is assumed to be greater than the other parallel surface

$$
\begin{aligned}
& \left.\frac{\partial \theta}{\partial x}\right|_{x=0}=0, \\
& \left.\frac{\partial \theta}{\partial x}\right|_{x=1}=0, \\
& \theta(x, 0)=s, \\
& \theta(x, \sigma)=s-1 .
\end{aligned}
$$

Two Isoflux and Two Isothermal Walls. Surfaces parallel to the $y$-axis are assumed isothermal

$$
\begin{aligned}
& \left.\frac{\partial \theta}{\partial y}\right|_{y=0}=-1, \\
& \left.\frac{\partial \theta}{\partial y}\right|_{y=\sigma}=1, \\
& \theta(0, y)=s \\
& \theta(1, y)=s,
\end{aligned}
$$

where $s=\left(T_{1}-T_{0}\right) /\left(T_{1}-T_{2}\right)$.

\section{Results and Discussion}

The dimensionless temperature equation (10) and the coupled velocity and microrotation equations $((7)-(9))$ together with the boundary conditions ((12a), (12b), (12c), (15a), (15b), (16a), and (16b)) are solved numerically using the finite difference method with 40 mesh points in both directions. Throughout the study unless specified otherwise the values $N=0.5, l=$ $1, m=3, \lambda=5, \mathrm{Ha}=3, s=-1.6$, and $\sigma=1$ are used. For validation, we use the following values: $N=0, \mathrm{Ha}=0$, $\lambda=-1$, and $\mathrm{Gr} / \mathrm{Re} \rightarrow 0$. Table 1 shows a comparison of the present values and those obtained by Barletta [12] and it can be seen that they are in in good agreement.

The coupling number $N$ characterizes the coupling of linear and rotational motion arising from the micromotion of the fluid molecules. $N$ signifies the coupling between the Newtonian $(\mu)$ and rotational viscosities $(\kappa)$ and hence $0 \leq$ $N<1$. For large values of $N$, the effect of microstructure becomes significant, whereas for small values of $N$ the individuality of the substructure is much less pronounced. As $\kappa \rightarrow 0$ (i.e., $N \rightarrow 0$ ), the micropolarity is lost and the fluid behaves as a nonpolar fluid. Hence, $N \rightarrow 0$ corresponds to a viscous fluid. More on this relation can be found. Detailed study comparing the classical and micropolar theory can be found in the work of Ansari et al. [28].

The results are discussed first for two of the sides (sides parallel to the $y$-axis) that are adiabatic and the others that are isothermal, that is, thermal boundary condition (Case 1). The profiles for velocity and microrotations $v_{1}$ and $v_{2}$ are presented in Figures 2(a)-2(e). From Figures $2(\mathrm{a})-2(\mathrm{c})$, it is observed that as the aspect ratio increases the flow reversal near the hot plate (plate with temperature $T_{1}$ ) increases. The profile of microrotation $v_{1}$ is antisymmetric, at $y=0.4$, whereas the microrotation component $v_{2}$ shows 


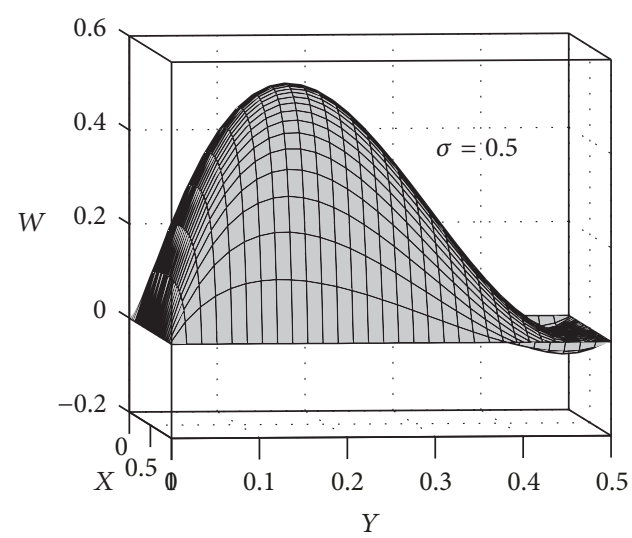

(a) Velocity profile

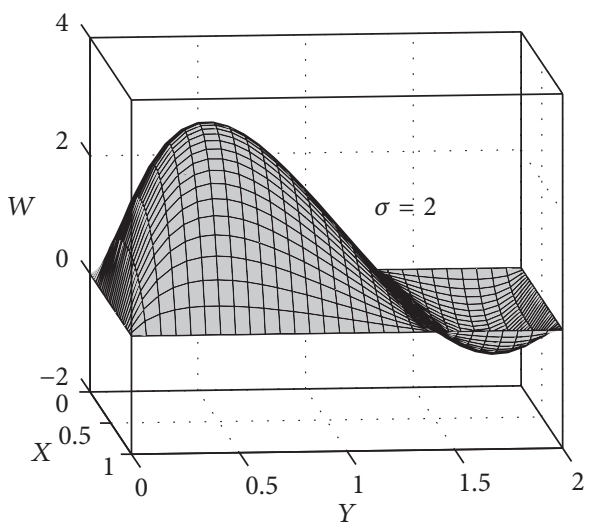

(c) Velocity profile

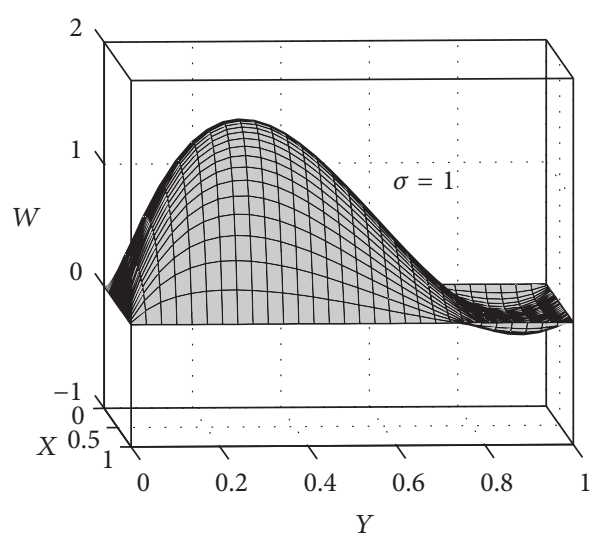

(b) Velocity profile

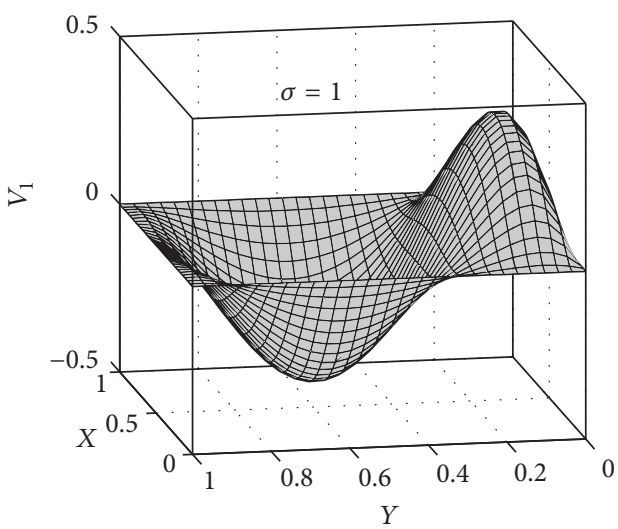

(d) Microrotation $V_{1}$

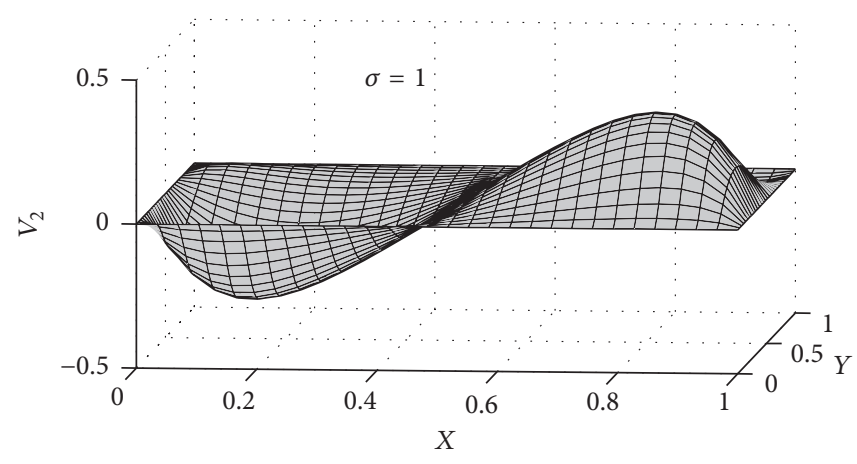

(e) Microrotation $V_{2}$

FIGURE 2: Case 1: different aspect ratios, with $\mathrm{Gr} / \mathrm{Re}=50$ and $S=0.5$.

antisymmetric rotation about the center for $\sigma=1$. It is also noted that microrotation components flow in opposite direction as it can be seen from Figures 2(d) and 2(e).

Figures 3(a)-3(c) show the effect of Gr/Re on velocity and microrotations for the aspect ratio $\sigma=1$. For $\mathrm{Gr} / \mathrm{Re}>0$, upward flow, when mean flow $U_{0}>0$, the velocity decreases with increase in $\mathrm{Gr} / \mathrm{Re}$ whereas the microrotations increase. It is also noted that the microrotation component $V_{2}$ shows a flow reversal near the plate as the value of $\mathrm{Gr} / \mathrm{Re}$ increases. The influence of the applied magnetic field is shown in Figure 3(d) for $\mathrm{Gr} / \mathrm{Re}=200$ and aspect ratio $\sigma=1$. It is seen that the presence of the magnetic field influences the flow behaviour at the center in addition to the decreasing nature when the value of Ha increases.

In the following discussion, we consider the boundary conditions where two of the sides are isothermal (sides parallel to the $y$-axis) and the other two sides are isoflux. The dimensionless temperature distribution (10) is solved independently of the velocity and microrotation from (10) and (16a) and (16b). The solution is shown in Figure 4(a). The profile of velocity and microrotations for different values of $\mathrm{Gr} / \mathrm{Re}$ for two isoflux and two isothermal walls is shown in Figures 4(b)-4(e). It is interesting to see that the flow reversals for velocity are dependent on the sign of $\mathrm{Gr} / \mathrm{Re}$ with respect 


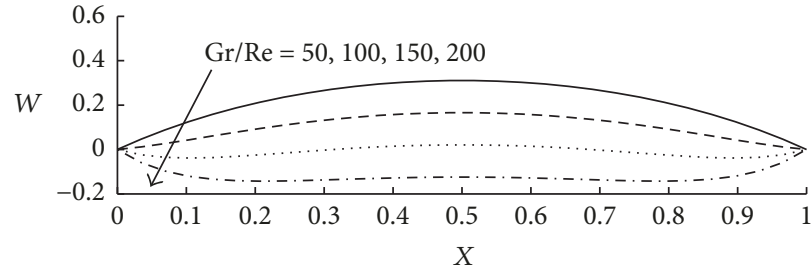

(a) The effect of $\mathrm{Gr} / \mathrm{Re}$ on velocity

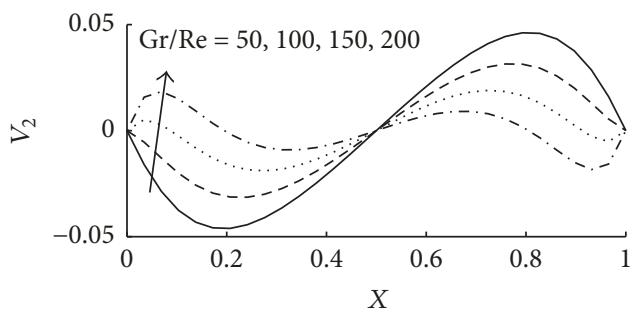

(c) The effect of $\mathrm{Gr} / \mathrm{Re}$ on microrotation $V_{2}$

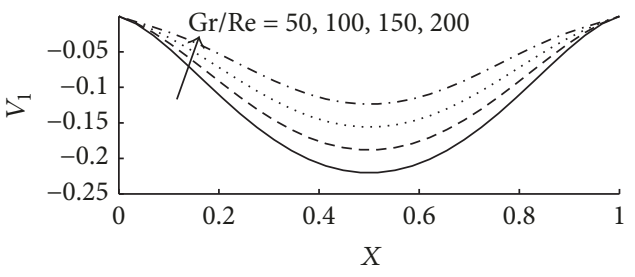

(b) The effect of $\mathrm{Gr} / \mathrm{Re}$ on microrotation $V_{1}$

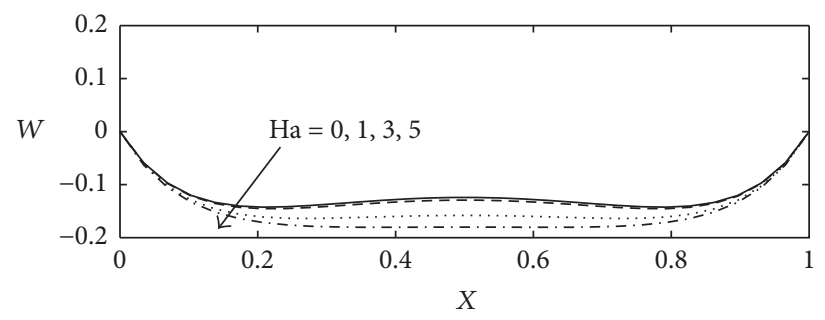

(d) The effect of Ha effect on velocity for $S=0.5$ for $\mathrm{Gr} / \mathrm{Re}=200$

FIGURE 3: Case 1: effects for thermal boundary condition.

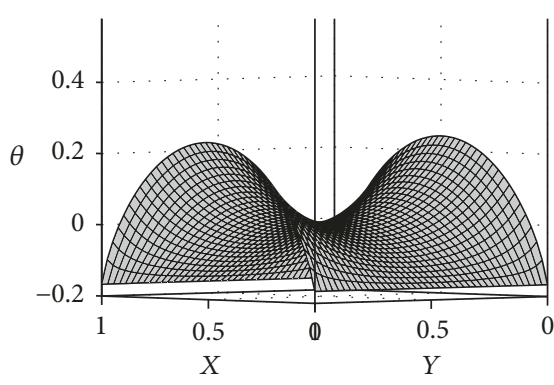

(a) Temperature profile

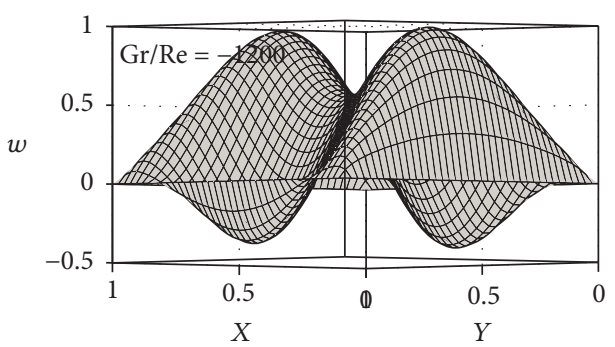

(c) Velocity profile

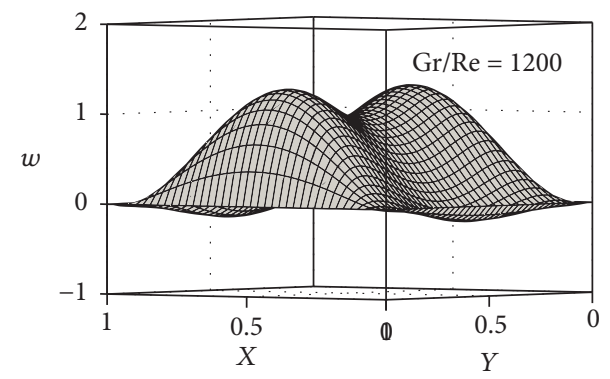

(b) Velocity profile

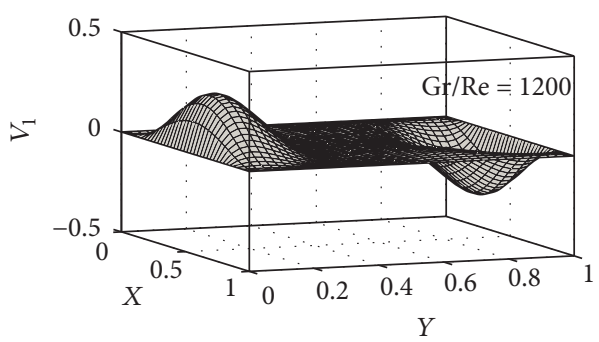

(d) Microrotation

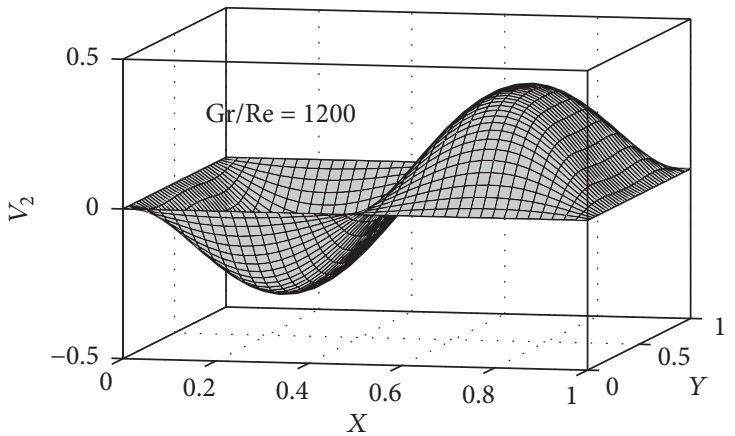

(e) Microrotation

Figure 4: Case 2: thermal boundary condition with different values of Gr/Re. 


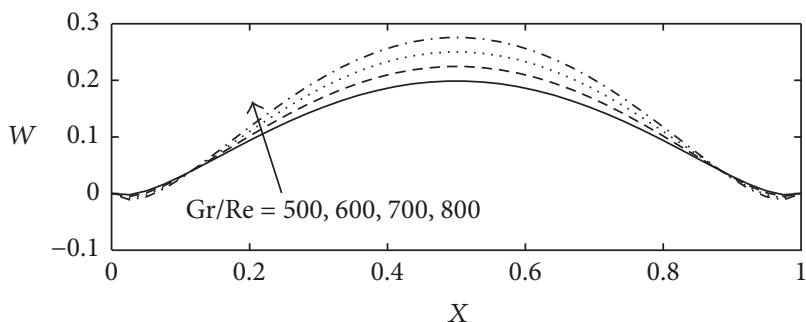

(a) Velocity

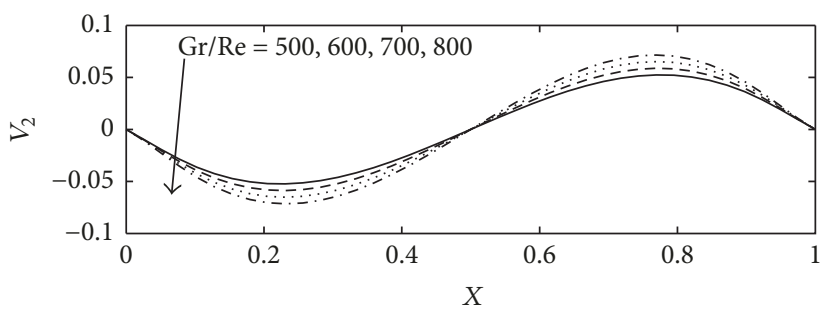

(c) Microrotation

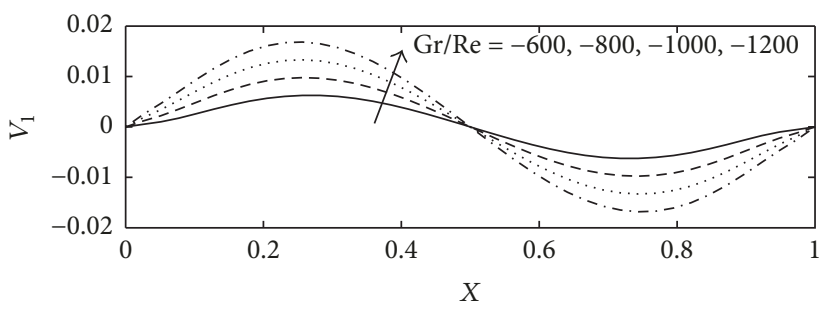

(e) Microrotation

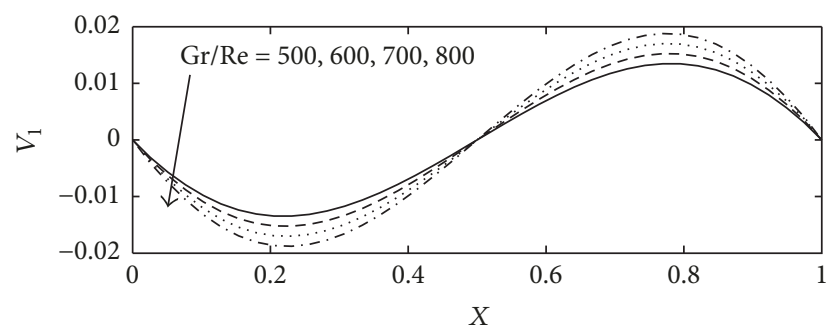

(b) Microrotation

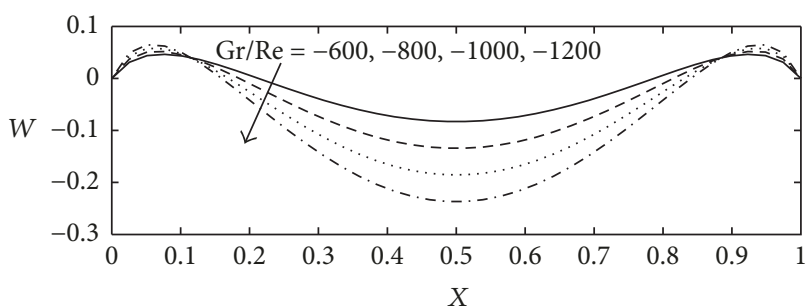

(d) Velocity

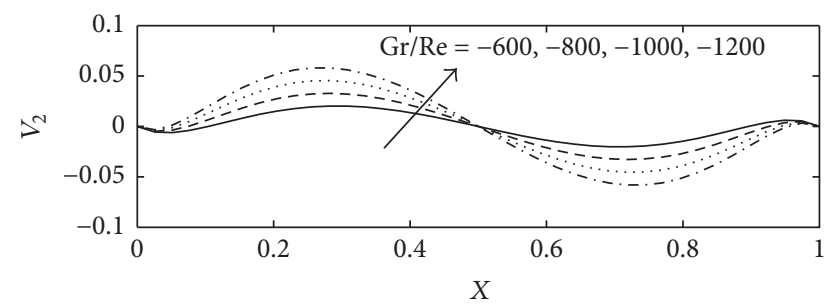

(f) Microrotation

FIGURE 5: Simulation of velocity and microrotation.

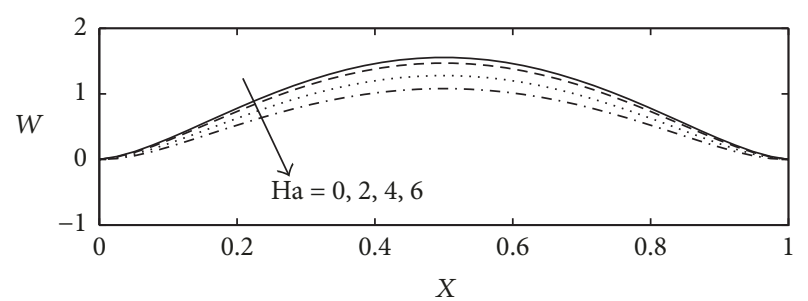

(a)

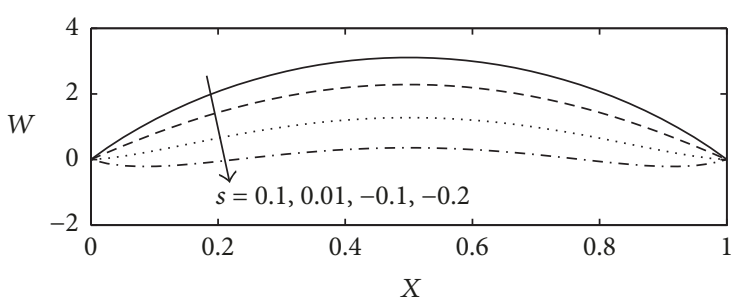

(b)

FIgURE 6: Simulation of velocity.

to the axes. It is also noted that microrotation $V_{1}$ is almost constant (no rotation) around the middle of the duct as seen in Figure 4(d).

For $\mathrm{Gr} / \mathrm{Re}>0$, the fluid is heating $\left(q_{w}>0\right)$ with upward mean flow; flow reversal is observed in the neighbourhood of the isothermal walls. Figures 5(a)-5(c) show that increasing the values of $\mathrm{Gr} / \mathrm{Re}$ decreases the velocity. In Figures 5(d)-5(f), flow reversal is observed for negative values of the parameter $\mathrm{Gr} / \mathrm{Re}$ next to the isoflux walls. Microrotation components show symmetric effects in general; it is also observed that the microrotation component $V_{2}$ shows reversal flow near the same plates.

The effect of the magnetic field is presented in Figure 6(b) which shows the magnetic field slows down the flow. It is also interesting to see that the higher the applied magnetic field in addition to the flow retardation, the more pronounced the flow reversal. In Figure 6(b), the effect of $s$ is presented. The numerical results show that when the value of $s$ is approximately $s>-0.1$, the commencement of flow reversal is observed for the aspect ratio $\sigma=1$, on a square duct.

\section{Conclusion}

A model has been developed for mixed convection micropolar fluid through a vertical rectangular duct with transverse magnetic field. Effects of different parameters governing the flow have been studied numerically. Two cases for the boundary conditions have been considered: the first case with two faces with constant different temperature and the others kept adiabatic and the other case with two faces 
isothermal and the others isoflux. Generally, increasing the aspect ratio shows more flow reversal. It is also observed that as $s \rightarrow-0.1$, the onset of flow reversal is observed. As the magnetic field increases, flow reversal increases and velocity decreases. For microrotation components, opposite direction flow is observed. It is also seen that, for the microrotation component $V_{1}$, the rotational effect is suction around the center of the duct for higher values of $\mathrm{Gr} / \mathrm{Re}$.

\section{Conflicts of Interest}

The authors declare that there are no conflicts of interest regarding the publication of this article.

\section{References}

[1] A. C. Eringen, Microcontinuum Field Theories I: Foundations and Solids, Springer-Verlag, New York, NY, USA, 1999.

[2] A. C. Eringen, Microcontinuum Field Theories-II: Fluent Media, Springer, New York, NY, USA, 2001.

[3] N. K. Axtell, M. Park, and J. H. Cushman, "Micromorphic fluid in an elastic porous body: blood flow in tissues with microcirculation," International Journal for Multiscale Computational Engineering, vol. 3, no. 1, pp. 71-84, 2005.

[4] R. Ansari, M. Bazdid-Vahdati, A. Shakouri, A. Norouzzadeh, and H. Rouhi, "Micromorphic first-order shear deformable plate element," Meccanica, vol. 51, no. 8, pp. 1797-1809, 2016.

[5] J. D. Lee and X. Wang, "Generalized micromorphic solids and fluids," International Journal of Engineering Science, vol. 49, no. 12, pp. 1378-1387, 2011.

[6] R. Ansari, M. Bazdid-Vahdati, A. H. Shakouri, A. Norouzzadeh, and H. Rouhi, "Micromorphic prism element," Mathematics and Mechanics of Solids, vol. 22, no. 6, pp. 1438-1461, 2017.

[7] A. C. Eringen, "Simple microfluids," International Journal of Engineering Science, vol. 2, pp. 205-217, 1964.

[8] A. C. Eringen, “Theory of micropolar fluids," Journal of Applied Mathematics and Mechanics, vol. 16, pp. 1-18, 1966.

[9] A. C. Eringen, "Theory of thermomicrofluids," Journal of Mathematical Analysis and Applications, vol. 38, no. 2, pp. 480-496, 1972.

[10] T. Ariman, N. D. Sylvester, and M. A. Turk, "Applications of microcontinuum fluid mechanics," International Journal of Engineering Science, vol. 12, no. 4, pp. 273-293, 1974.

[11] V. A. Eremeyev, L. P. Lebedev, and H. Altenbach, Foundations of Micropolar Mechanics, Springer Sci and Business Media, 2013.

[12] A. Barletta, "Analysis of flow reversal for laminar mixed convection in a vertical rectangular duct with one or more isothermal walls," International Journal of Heat and Mass Transfer, vol. 44, no. 18, pp. 3481-3497, 2001.

[13] S. E. Ahmed, "Numerical study of MHD natural convection in an inclined rectangular cavity with internal heat generation filled with a porous medium under the influence of joule heating," Latin American Applied Research, vol. 43, pp. 67-71, 2013.

[14] M. Spiga and G. L. Morino, "A symmetric solution for velocity profile in laminar flow through rectangular ducts," International Communications in Heat and Mass Transfer, vol. 21, no. 4, pp. 469-475, 1994.

[15] D. Srnivasacharya and M. Shiferaw, "MHD flow of micropolar fluid in a rectangular duct with hall and ion slip effects," Journal of the Brazilian Society of Mechanical Sciences and Engineering, vol. 4, no. 313, 2008.

[16] H. H. S. Chu, S. W. Churchill, and C. V. S. Patterson, "The effect of heater size, location, aspect ratio, and boundary conditions on two-dimensional, laminar, natural convection in rectangular channels," Journal of Heat Transfer, vol. 98, 1976.

[17] M. E. Sayed-Ahmed and H. A. Attia, "The effect of Hall current on magnetohydrodynamic flow and heat transfer for Bingham fluids in a rectangular duct," Canadian Journal of Physics, vol. 83, no. 6, pp. 637-651, 2005.

[18] F. C. Chou and G. J. Hwang, "Vorticity-velocity method for Graetz problem with the effect of natural convection in a horizontal rectangular channel with uniform wall heat flux," Journal of Heat Transfer, vol. 109, pp. 704-710, 1987.

[19] K. Ramakrishna, S. G. Rubin, and P. K. Khosla, "Laminar natural convection along vertical square ducts, Numer," Heat Transfer, vol. 5, pp. 59-79, 1982.

[20] K. C. Cheng and J. W. Ou, "Convection instability and finite amplitude convection in the entrance region of horizontal rectangular channels heated from below," Proceedings of 7 th International Heat Transfer Conference, vol. 2, pp. 189-194, 1982.

[21] W. Aung and G. Worku, "Mixed convection in ducts with asymmetric wall heat fluxes," ASME Journal of Heat Transfer, vol. 109, pp. 947-951, 1987.

[22] H. V. Mahaney, F. P. Incropera, and S. Ramadhyani, "Development of laminar mixed convection flow in a horizontal rectangular duct with uniform bottom heating," Numerical Heat Transfer, Part B: Fundamentals, vol. 12, no. 2, pp. 137-155, 1987.

[23] M. M. Abou-Ellail and S. M. Morcos, "Buoyancy effects in the entrance region of horizontal rectangular channels," Journal of Heat Transfer, vol. 105, pp. 924-928, 1983.

[24] C.-C. Huang, W.-M. Yan, and J.-H. Jang, "Laminar mixed convection heat and mass transfer in vertical rectangular ducts with film evaporation and condensation," International Journal of Heat and Mass Transfer, vol. 48, no. 9, pp. 1772-1784, 2005.

[25] W. M. Yan and T. F. Lin, "Effects of wetted wall on laminar mixed convection in a vertical channel," Journal of Thermophysics and Heat Transfer, vol. 3, no. 1, pp. 94-96, 1989.

[26] W.-M. Yan, "Turbulent mixed convection heat and mass transfer in a wetted channel," Journal of Heat Transfer, vol. 117, no. 1, pp. 229-233, 1995.

[27] T. Hayat, S. Nadeem, and S. Asghar, "Hydromagnetic Couette flow of an Oldroyd-B fluid in a rotating system," International Journal of Engineering Science, vol. 42, no. 1, pp. 65-78, 2004.

[28] R. Ansari, A. H. Shakouri, M. Bazdid-Vahdati, A. Norouzzadeh, and H. Rouhi, "A nonclassical finite element approach for the nonlinear analysis of micropolar plates," Journal of Computational and Nonlinear Dynamics, vol. 12, no. 1, Article ID 011019, 2017. 


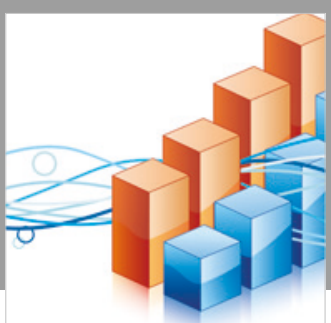

Advances in

Operations Research

\section{-n-m}
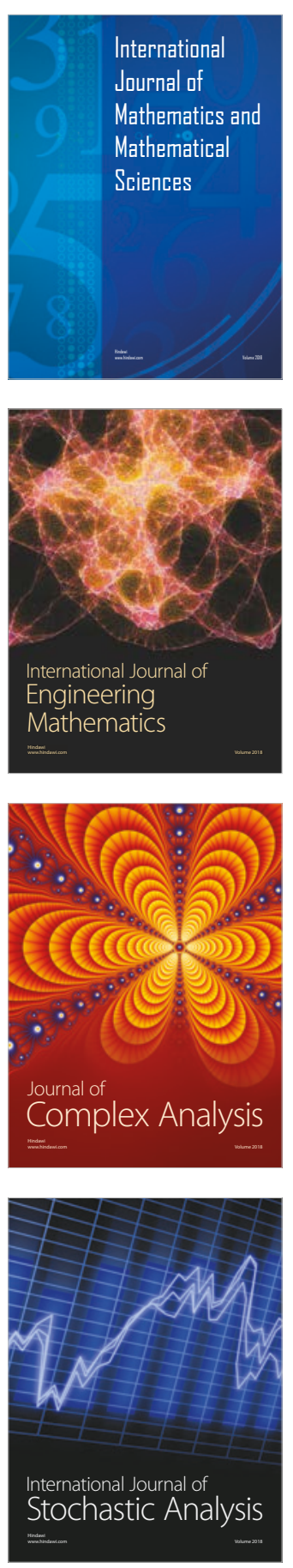
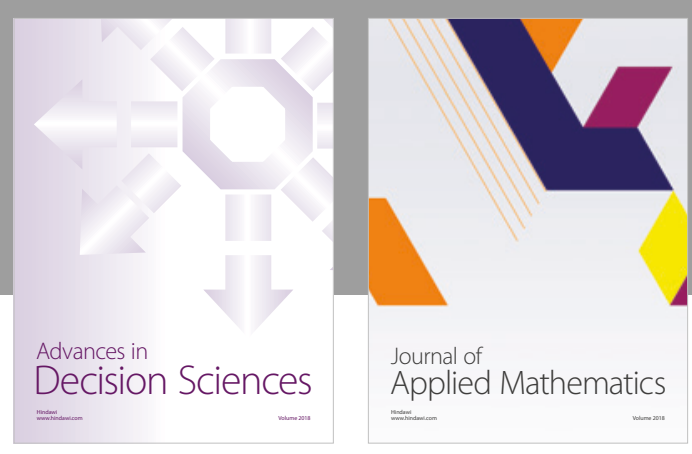

Journal of

Applied Mathematics
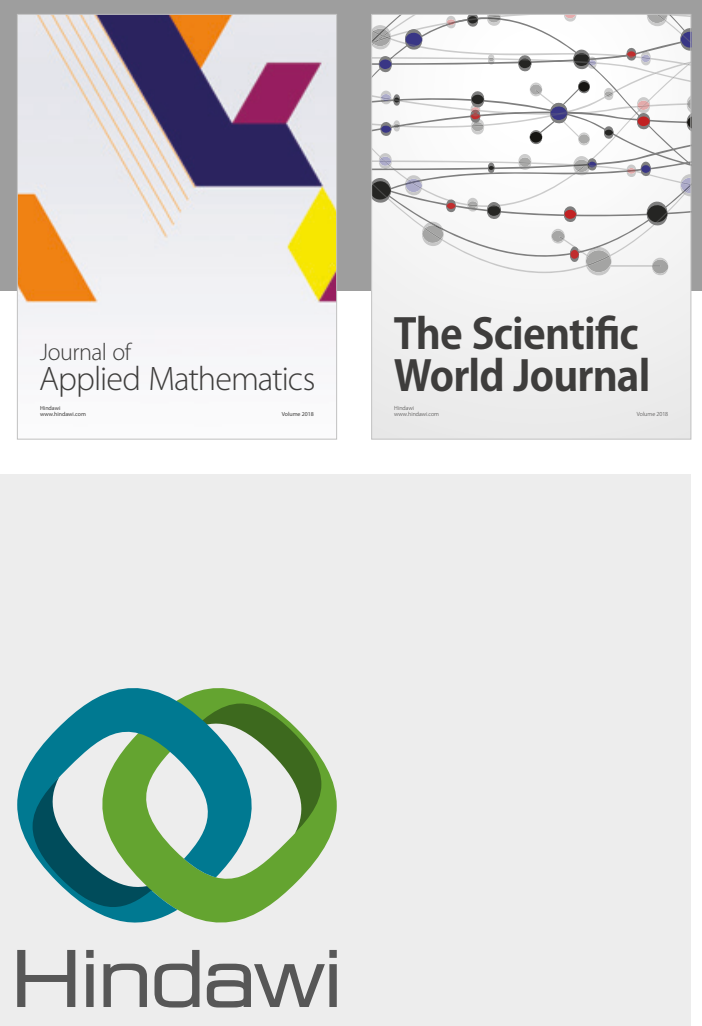

Submit your manuscripts at

www.hindawi.com

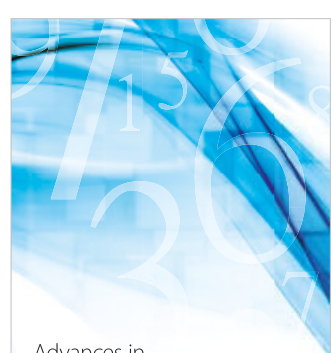

Advances in
Numerical Analysis
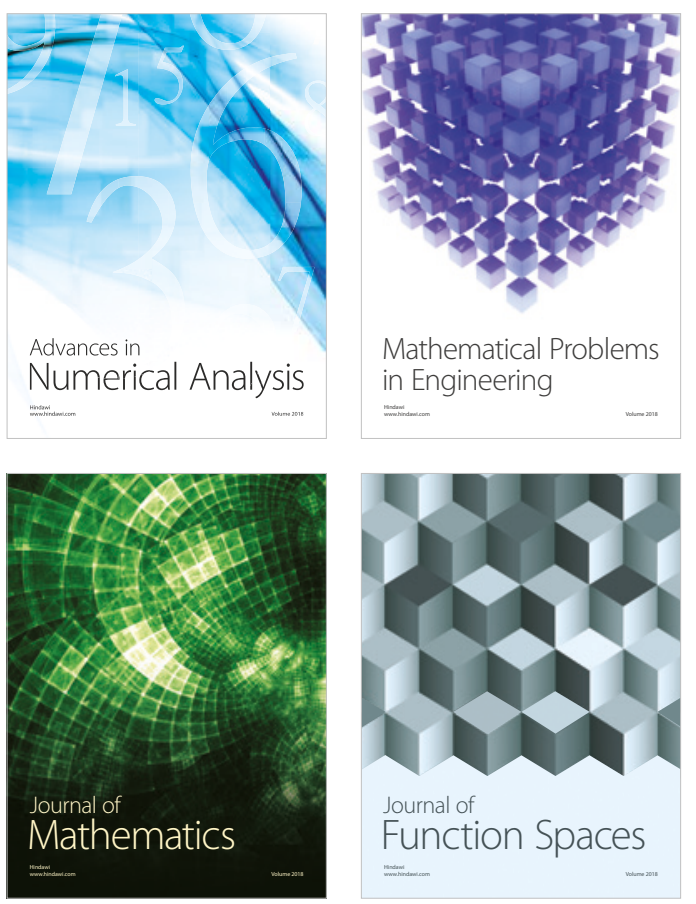

Mathematical Problems in Engineering

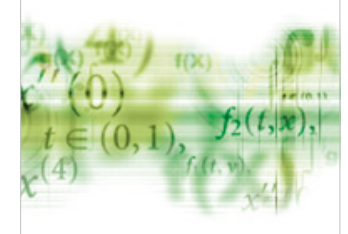

International Journal of

Differential Equations

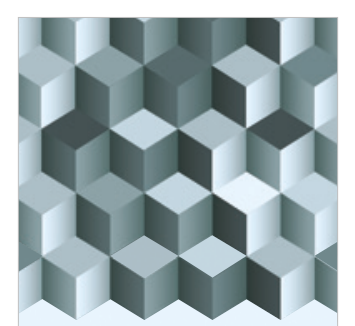

Journal of

Function Spaces

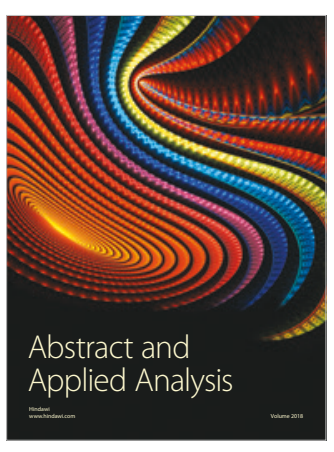

The Scientific

World Journal

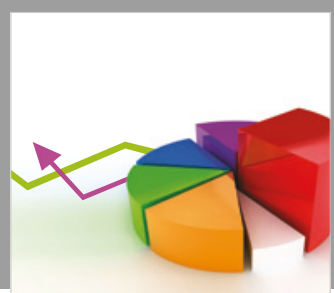

Journal of

Probability and Statistics
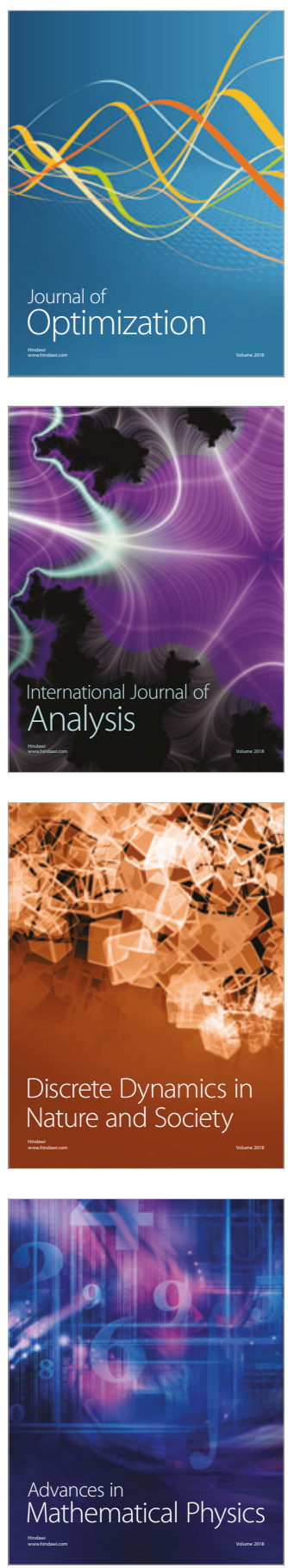